\title{
Antiviral Therapy in Cytomegalovirus-Associated Biliary Atresia
}

\author{
Praveen Mathur ${ }^{1} \cdot$ Priyanka Udawat $^{2,3}$. Priyanshu Mathur ${ }^{4}$ - Dilip Ramrakhiani ${ }^{5}$ - Sandeep K. Mathur ${ }^{6}$. \\ S. Sitaraman ${ }^{4}$. R. K. Gupta ${ }^{4}$ Saurav Sultania ${ }^{1} \cdot$ Reshu Gupta ${ }^{3}$
}

Received: 21 September 2021 / Accepted: 11 January 2022/Published online: 8 March 2022

c) Dr. K C Chaudhuri Foundation 2022

To the Editor: Biliary atresia (BA) is characterized by progressive inflammation and fibrosis of both the extrahepatic and intrahepatic bile ducts within 3 mo of life [1,2]. Antiviral drugs such as ganciclovir or its oral prodrug valganciclovir have been in use for the treatment of congenital CMV infection, but no standard treatment protocol exist for $\mathrm{CMV}+$ ve BA $[3,4]$.

This study involves $22 \mathrm{CMV}$-positive and $33 \mathrm{CMV}$-negative patients. All CMV-positive patients were started on IV ganciclovir $(6 \mathrm{mg} / \mathrm{kg} / \mathrm{dose} \mathrm{BD})$, soon after the finding of a positive urinary PCR for CMV, which was followed by tab valganciclo$\operatorname{vir}(16 \mathrm{mg} / \mathrm{kg} / \mathrm{day}$ BD for $3 \mathrm{wk})$. Successful outcome following KPE was defined by clearance of jaundice $(\leq 2 \mathrm{mg} / \mathrm{dL})$ after 6 mo of surgery.

CMV-positive BA infants were older at their first consultation than CMV-negative BA infants, and showed higher S. bilirubin, higher degree of inflammation, and fibrosis in comparison to CMV-negative BA (Supplementary Table S1). Jaundice clearance after 6 mo of surgery was $80 \%$ in the AVT-treated CMV-positive group, while it was $62 \%$ in the CMV-negative group (Supplementary File 2). However, we did not observe any significant difference in the overall survival between the two groups. These results suggest the

Reshu Gupta

scienceadv13@gmail.com

1 Department of Pediatric Surgery, SMS Medical College \& Attached Hospitals, Jaipur, Rajasthan, India

2 Present Address: Institute of Digestive \& Liver Care, S. L. Raheja Hospital, Mumbai, Maharashtra, India

3 Department of Pediatric Medicine, SMS Medical College \& Attached Hospitals, Jaipur, Rajasthan 302004, India

4 Department of Pediatric Medicine, SMS Medical College, Jaipur, Rajasthan, India

5 Department of GI \& Liver Pathology, SMS Medical College \& Attached Hospitals, Jaipur, Rajasthan, India

6 Department of Endocrinology \& Molecular Genetic Research Lab, SMS Medical College, Jaipur, Rajasthan, India positive outcome of AVT, providing hope to an otherwise dreary disease subset. For effective therapy and timely initiation of treatment in cases of CMV-associated BA, we need to refine our understanding of the etiology of this subset of disease. The treatment regime of using the antiviral drugs in both the pre- and postsurgical period seems to be a better option, as evident by the encouragingly high number of jaundice clearance rates in our series. Therefore, there is an urgent need to find an effective treatment targeting the virus to keep this disease in check, if not cure it.

Supplementary Information The online version contains supplementary material available at https://doi.org/10.1007/s12098-022-04093-4.

\section{Declarations}

Conflict of Interest None.

\section{References}

1. Sanghai SR, Shah I, Bhatnagar S, Murthy A. Incidence and prognostic factors associated with biliary atresia in western India. Ann Hepatol. 2009;8:120-2.

2. Burns J, Davenport M. Adjuvant treatments for biliary atresia. Transl Pediatr. 2020;9:253-65.

3. Shah I, Bhatnagar S. Biliary atresia with cytomegalovirus infection and its response to ganciclovir. Trop Gastroenterol. 2014;35:56-8.

4. Parolini F, Hadzic N, Davenport M. Adjuvant therapy of cytomegalovirus IgM+ve associated biliary atresia: prima facie evidence of effect. J Pediatr Surg. 2019;54:1941-5.

Publisher's Note Springer Nature remains neutral with regard to jurisdictional claims in published maps and institutional affiliations. 